Please do not remove this page

RMIT

UNIVERSITY

\title{
Strategies to identify changes in SEMG due to muscle fatigue during cycling
}

Kumar, Dinesh; Singh, Vijay Pal; Cosic, Irena; Giudice, S; Hawley, John

https://researchrepository.rmit.edu.au/esploro/outputs/9921861699701341/filesAndLinks?institution=61RMIT_INST\&index=null

Kumar, D., Singh, V. P., Cosic, I., Giudice, S., \& Hawley, J. (2005). Strategies to identify changes in SEMG due to muscle fatigue during cycling. Proceedings of the 27th Annual International Conference of the IEEE Engineering in Medicine and Biology Society. https://doi.org/10.1109/IEMBS.2005.1616036

Published Version: https://doi.org/10.1109/IEMBS.2005.1616036

Repository homepage: https://researchrepository.rmit.edu.au

(c) 2005 IEEE. Personal use of this material is permitted. However, permission to reprint/republish this material for advertising or promotional purposes or for creating new collective works for resale or redistribution to servers or lists, or to reuse any copyrighted component of this work in other works must be obtained from the IEEE.

Downloaded On 2023/04/26 19:57:20 +1000 


\title{
Strategies to identify changes in SEMG due to muscle fatigue during cycling
}

\author{
D K Kumar, V P Singh, I Cosic, S L Giudice and J Hawley
}

\begin{abstract}
Detection, quantification and analysis of muscle fatigue is crucial in occupational/rehabilitation and sporting settings. Sports organizations such as Australian Institute of Sports (AIS) currently monitor fatigue by a battery of tests including invasive techniques that require taking blood samples and/or muscle biopsies, the latter of which is highly invasive, painful, time consuming and expensive. SEMG is non-invasive monitoring of the muscle activation and is an indication of localized muscle fatigue based on the observed shift of the power spectral density of the SEMG. But the success of SEMG based techniques is currently limited to isometric contraction and is not acceptable to the human movement community. This paper proposes and tests a simple signal processing technique to identify the onset of muscle fatigue during cyclic activities of muscles such as VL and VM during cycling. Based on experiments conducted with 7 participants, using power output as a measure of fatigue, the technique is able to identify the muscle fatigue with $98 \%$ significance.
\end{abstract}

\section{INTRODUCTION}

Enhancement of human performance is important for improvement of quality of life, for sports and for industry. For this purpose, it is essential to be able to determine the individual threshold at which the onset of fatigue occurs. Fatigue is the point at which the muscle is no longer able to sustain the required force or power output. Detection, quantification and analysis of muscle fatigue is crucial in occupational/rehabilitation and sporting settings. Undetected muscle fatigue can cause injury to the subject, and besides the pain and suffering that occurs as a consequence of injury, injury and its sequel are a financial burden to industry and society. Sports organizations such as Australian Institute of Sports (AIS) currently monitor fatigue by a battery of tests including invasive techniques that require taking blood samples and/or muscle biopsies, the latter of which is highly invasive, painful, time consuming and expensive.

The development of muscle fatigue during exercise is associated with a decrement in performance. Mechanisms of muscle fatigue depend on the exercise conditions (eg. duration and intensity) and the subject's level of physical fitness. The decrements in skeletal muscle power output is also related to neural drive reductions may also lead to

\footnotetext{
Manuscript received on July 15, 2005.

DK Kumar, VP Singh, I Cosic are with School of Electrical and Computer Engineering, RMIT University, Melbourne VIC 3000 Australia. (Email: dinesh@ @rmit.edu.au)
}

SL Giudice and J Hawley are with Medical Sciences, RMIT University, Bundoora, VIC 3083 Australia

(Email: john.hawley@rmit.edu.au) muscle fatigue in prolonged exercise [8]. This leads to the use of electromyogram- a measure of the electrical activity of the muscle - as being an indicator of muscle fatigue.

Invasive markers of high intensity fatigue include depletion of high-energy phosphates including creatine phosphate, accumulation of metabolic bi products including [lactate], $\left[\mathrm{H}^{+}\right]$and inorganic phosphate [7]. Altered SR Ca ${ }^{2+}$ ATPase and $\mathrm{Na}^{+}, \mathrm{K}^{+}$-ATPase activity have also been implicated in the fatigue process [14]. Fatigue during prolonged exercise leading to fatigue in 2-3 hours has been associated with depletion of muscle glycogen stores [6]. These measurements are valid and reliable indicators of fatigue but are invasive, and not practical outside the laboratory setting.

Some researchers have attempted to use the electrical activity of muscle and muscle activation using surface electromyogram (SEMG) to study fatigue using surface $[1$, 3 , and 11]. SEMG is a result of summation of number of separate motor unit action potential in muscles and is dependent on numerous factors such as the rate of stimulation of the muscle, size of motor units recruited, morphology of the motor units, electrical properties of the tissues and the presence of any synchronisation of the activity of different motor units. The rate of stimulation of the muscle and size of active motor units is dependent on the force of contraction required to be produced by the muscle. It is a complex and non-stationary signal with large inter and intra subject variations.

Research analysis to date aimed at extracting from the SEMG an indication of localized muscle fatigue has been based on the observed shift of the power spectral density of the SEMG [2, 10]. When the muscle is fatigued, a strengthening of low-frequency components and a reduction in intensity of high-frequency components modifies the spectrum of the SEMG signal. Several parametric measures of SEMG signal have been used as a relative indicator of the muscle fatigue phenomenon for an individual subject. These include the Root Mean Square (RMS), spectrum analysis (instantaneous, mean and median frequency) and zero crossing rates. The authors have used a combination of wavelets and neural networks to reliably classify SEMG with isometric muscle contraction status [5] - a technique that is more reliable for identifying the onset of muscle fatigue [4].

When the muscle is contracting to produce motion, the strength of contraction is varying and variation in SEMG is far greater than during isometric contraction. Muscle fatigue may occur due to isometric or non-isometric muscle contraction and may be due to low, high or very high level 
contraction and hence it is important that SEMG be able to identify the onset of muscle fatigue in both the conditions. The contraction may be due to aerobic or anaerobic origin [7]. The biochemical processes associated with the onset of muscle fatigue changes due to these different causes are believed to be different [12]. This makes the interpretation of SEMG signals from dynamic contractions much more difficult [9]. The success of the current techniques is limited to isometric contraction [4] and is not acceptable to the human movement community This paper reports a novel technique with the aim at determining the change of SEMG properties due to the onset of localised muscle fatigue during dynamic cyclic motion at high levels of contraction (30 seconds sprint). Power output by the athlete was used as the gold standard to identify the onset of muscle fatigue. The outcomes from this research would be to validate (via objective invasive procedures) the use of a novel, noninvasive marker (SEMG) for muscle fatigue to be utilised in industrial, rehabilitation and sporting situations.

\section{THEORY}

It has been well established that during isometric contraction, there is a shift in the spectrum of the recorded SEMG due to muscle fatigue. Studies by other researchers and experiments conducted by our team have determined that during cycling, the vastus lateralis (VL) and vastus medialis (VM) muscle undergoes cyclic contraction and the properties of SEMG vary cyclically from zero to maximum. Due to such variation in the signal, it is essential to establish a reference in a cycle for the purpose of determining changes.

The proposed technique requires computing the envelope of the signal, determining the peak activity during the cycles and computing the spectrum using FFT for a 100 milliseconds window of the signal. This corresponds to 20 $\mathrm{Hz}$ lower end frequency and the duration corresponds to 45 degrees of the cycle and the region of maximal contraction of the muscles and hence was the most suitable compromise. To overcome the difficulty due to inter-subject variation, ratio of the median frequency and RMS under pre and post fatigue conditions has been considered to identify the change. This paper also reports the use of markers that determine the angular location in each cycle for correlating the muscle activity with this location. This paper reports the identification of the onset of muscle fatigue by the drop in power output and reduction in speed of cycling.

\section{METHODS/ RESEARCH PROCEDURE}

Seven (7) moderately active male volunteer participants were recruited (age ranging between 18-40 years) and were medically screened. Written consent was signed by them before participating in the study.

A standardized $30 \mathrm{sec}$ cycle test to fatigue on a Lode ergo-meter with customized software for sprint measurements was conducted. Power output was measured along with SEMG. The subject was termed as 'fatigued' when the power output drops by greater than $33 \%$.
The skin was lightly abraded using disposable skin defoliator and cleaned with a swab soaked in alcohol to reduce skin impedance to less than $60 \mathrm{~K} \Omega$. Heart-rate was monitored (Polar, Finland) to ensure safety of the participant. SEMG was recorded from the three channels (Table 1) using Delysis (USA) SEMG recording system with fixed inter-electrode distance.

Windowed RMS was computed to identify the envelopes and bursts of activity. Three cycles near the start and near the end of the exercise (pre and post fatigue conditions) were considered. The peak of each cycle was identified based on moving root mean square (MRMS), and a small section (100 milliseconds) of the raw SEMG data were analyzed (figure 1). The first and the last cycle of the recordings were discarded because of sudden changes taking place during these segments. Signal processing was done using MATLAB software package.

TABLE 1

CHANNEL ASSIGNMENT FOR DIFFERENT MUSCLES

\begin{tabular}{|l|l|}
\hline Channel 1 & Vastus lateralis (outside thigh muscle - front) \\
\hline Channel 2 & Vastus medialis (inside thigh muscle - front) \\
\hline Channel 3 & Rectus femoris (middle thigh muscle - front) \\
\hline Channel 4 & Medial gastrocnemius (inside calf muscle) \\
\hline Channel 5 & Lateral gastrocnemius (outside calf muscle)
\end{tabular}

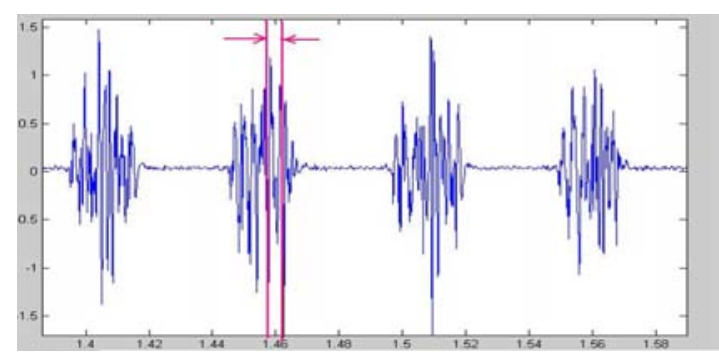

Figure 1. Windowed Raw Signal (Illustration only)

The RMS and median frequency of each of the three envelopes representing the post and pre fatigue conditions were computed. An average was computed for pre and post fatigue condition. Using this, ratio of the pre and post RMS and MF was computed for each subject and for the five channels. A ratio less than one would indicate a decrease due to fatigue. The results were statistically evaluated to determine the significance.

\section{RESULTS}

Figure 3 plots the ratio of normalized RMS of the windowed signal under pre and post fatigue conditions while figure 4 plots the ratio of normalized median frequency of the windowed signal under pre and post fatigue conditions. The power output of the athlete at the cycle wheel for the duration of the sprint is plotted in figure 5. Variation of the speed of cycling over the duration of the sprint is plotted in figure 2 . 
Table 2

$\mathrm{T}$ Test for median frequency and RMS

\begin{tabular}{|c|c|c|c|c|c|c|}
\hline & \multicolumn{2}{|c|}{ Median Frequency } & \multicolumn{3}{c|}{ RMS } \\
\hline $\begin{array}{c}\text { Chan } \\
\text { nel }\end{array}$ & $\mathrm{T}$ & $\mathrm{P}$ & $\begin{array}{c}\text { Signifi } \\
\text { cance }\end{array}$ & $\mathrm{T}$ & $\mathrm{P}$ & $\begin{array}{c}\text { Signif } \\
\text { icance }\end{array}$ \\
\hline 1 & 3.07 & 0.01 & $95 \%$ & 0.37 & 0.36 & $28 \%$ \\
\hline 2 & 3.19 & 0.01 & $98 \%$ & 0.67 & 0.73 & $74 \%$ \\
\hline 3 & 2.05 & 0.04 & $91 \%$ & 0.49 & 0.68 & $69 \%$ \\
\hline 4 & 1.9 & 0.05 & $90 \%$ & 2.47 & 0.02 & 95 \\
\hline 5 & 2.6 & 0.02 & 96 & 2.64 & 0.019 & 96 \\
\hline
\end{tabular}

\section{OBSERVATIONS AND DISCUSSIONS}

From figure 3 it is observed that there is little change in RMS of SEMG pre and post muscle fatigue. From figure 4 it is observed that there is a drop of about $20 \%$ of median frequency for all the five channels. From figure 5 it is evident that the power maximum occurs at the end of the first quarter followed by a gradual decline and the final power output is approximately half of the power at the start.

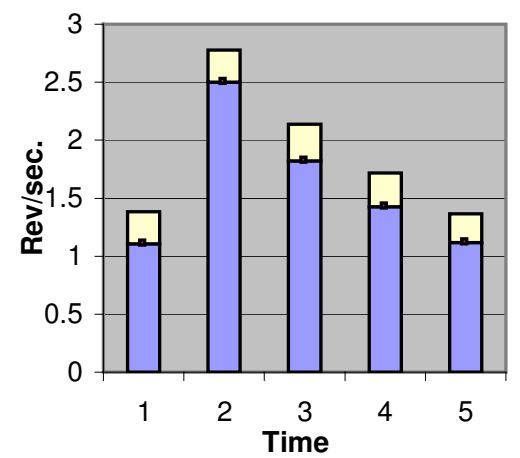

Fig 2. Variation in the speed of cycling

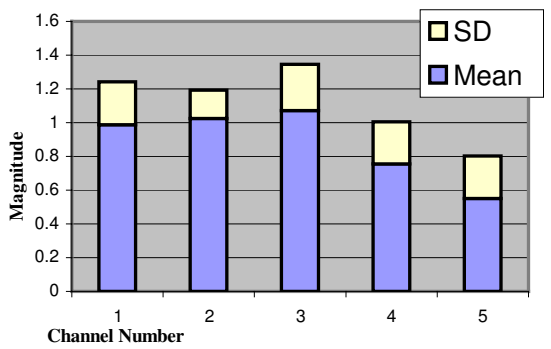

Fig 3. Normalized RMS

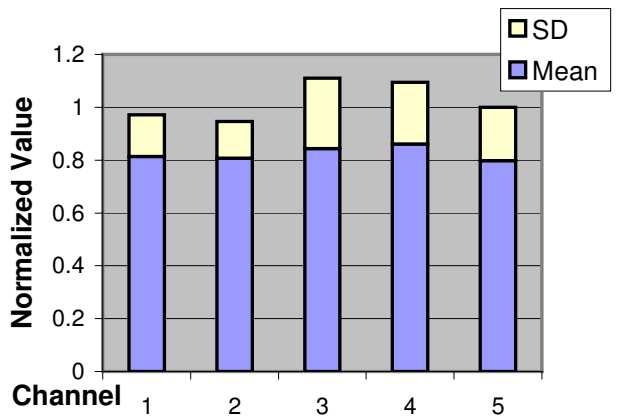

Fig 4. Normalized Frequency

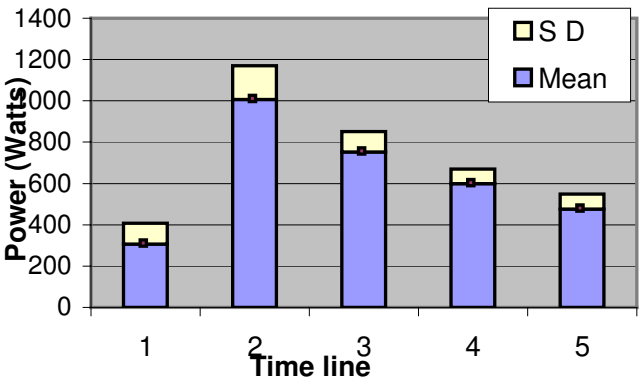

Figure 5. Power Output

Similar is the trend in the speed of cycling as this can be noticed from figure 2. Some of the specific observations and subsequent conclusions are::

(i) There is a significant $(90 \%+)$ decline in the median frequency of all the channels by an approximate $20 \%$ with (table 2) suggesting the use of median frequency ratio as a useful measure of muscle fatigue during maximal exercise.

(ii) There is no significant change in the RMS of channel 1, 2 and 3 (less than 75\%). This suggests that magnitude of SEMG is not significantly affected by the fatiguing of the muscles during dynamic activity.

(iii) There is a significant reduction of power output by the cyclist with average reduction greater than 50\%. This suggests that in all cases, there was significant muscle fatigue due to the 30 seconds fixed load sprint.

(iv) Visual inspection suggests that the reduction in power is directly related to reduction in speed of cycling. Thus it appears that muscle fatigue affects the speed of cycling which results in reduction in power output.

\section{REFERENCE}

[1] A V Nargol, A P Jones, P J Kelly, C Greenough, "Factors in the reproducibility of electromyographic power spectrum analysis of lumbar paraspinal muscle fatigue" Spine , 1999 vol. 24 issue 9 , pp. 883-888 
[2] C J De Luca, "Myoelectric manifestation of localized muscle fatigue in humans", CRC critical review in Biomedical Engineering, vol.11, issue 4, pp 251-279

[3] C Oliver, K Tillostson, A P Jones, R Royal "Reproducibility of lumbar paraspinal surface electromyogram power spectra" Clin. Biomech., 1996 Vol. 11, issue 6, pp. 317-321

[4] D Kumar, N Pah, and A Bradley. "Wavelet Analysis of Surface Electromyography to Determine Muscle Fatigue", IEEE transactions on neural systems and rehabilitation engineering, 2003, vol. 11, no. 4

[5] D Kumar, N Pah "Neural Networks and Wavelets for EMG analysis". J EMG and Clinical Neuro, 2001 Vol. 40, issue 7 pp. 411- 421

[6] E Coyle, A Coggan, M Hemmert, "Muscle glycogen utilization during prolonged strenuous exercise when fed carbohydrate" $\mathrm{J}$ App Physio 1986. vol 61, issue 1, pp.165-72

[7] G C Bogdanis, M E Nevill, H K Lakomy, L H Boobis, "Power output and muscle metabolism during and following recovery from 10 and $20 \mathrm{~s}$ of maximal sprint exercise in humans". Acta Physiol Scand, 1998. Vol. 163, 261-72

[8] G Kamen, G Caldwell. "Physiology and Interpretation of the Electromyogram", Journal of Neurophysiology, 1996, vol. 13,pp. 366-384,

[9] J Potvin. "Effects of muscle kinematics on surface EMG amplitude and frequency during fatiguing dynamic contractions", 1997 J Appl Physiol, vol. 82, pp.144-151

[10] J R Cram, G S Kasman, and J Holtz, "Introduction to Surface Electromyography", 1998 Aspen Publishers, Gaithersburg, Maryland

[11]N Pah, D Kumar and P Burton, "Adding Wavelet Decomposition to Neural Networks for the Classification of Fatigue SEMG", 2nd Conference of the Victorian chapter of the IEEE engineering in medicine and biology society, February 2001

[12] R H Fitts. 'Cellular mechanisms of muscle fatigue'. Physiol Rev., 1994 Vol.74, issue 1, pp. 49-94

[13] S F Fraser, J L Li, M F Carey, X N Wang, X.N., Sangkabutra, T., Sostaric, S., Selig, K Kjeldsen, and M McKenna. "Fatigue depresses maximal in-vitro skeletal muscle $\mathrm{Na}^{+}, \mathrm{K}^{+}$-ATPase activity in untrained and trained individuals". J. Appl. Physio; 2002 vol 93 pp. $1650-1659$ 